\title{
Development and evaluation of a microcomputer testing terminal for testing and instruction
}

\author{
LYLE R. McKNIGHT, BRIAN K. WATERS, and JOSEPH P. LAMOS \\ Air Force Human Resources Laboratory, Lowry Air Force Base, Colorado 80230
}

\begin{abstract}
This paper describes the development and evaluation of the hardware and software of a stand-alone microcomputerized testing terminal. The microterminal represents a viable way of conducting and scoring multiple-choice tests and reliably obtains this information for presentation to a centralized management computer system. Obligating informational presentation to regular programmed materials and eliminating the need to handle constructed responses removes the requirements for large display features, thus contributing greatly to the low-cost feature of the microterminal.
\end{abstract}

The Technical Training Division of the Air Force Human Resources Laboratory (AFHRL) at Lowry AFB, Colorado, is currently involved in the development of a large computer-based instructional system, the Advanced Instructional System (AIS). The AIS is designed to handle a daily load of up to 2,100 students within four Air Force technical training courses. As a computer-based system, the AIS provides primarily computer-managed instruction (CMI). In this context, computer-assisted instruction (CAI) is one of many pedagogical approaches that can be used in the AIS, but due to present high terminal costs use has been limited.

Cost, of course, is a major factor in the implementation of a computer-based instructional (CBI) system. Any CBI system, if it is to maintain cost effectiveness, must be sensitive to the costs of courseware (instructional materials), software, and the capital investment in hardware. Hardware costs associated with a CAI system can be greatly decreased with the aid of microprocessor technology. This low-cost option is, however, conditional on the fact that one is willing to accept a device that does not incorporate a "do everything" capability, but rather depends on building in those capabilities necessary and peculiar to one's own special requirements. Our design, centering around the use of a microprocessor, and in part deriving its name from this technology, has been labeled a "microterminal."

The microterminal, its dynamic response-handling capability combined with the cost-effective presentation of information provided by programmed texts or audiovisual materials, begins to fill the void of instructional requirements and computer usage which lies between the more traditional conceptions of CMI and CAI.

The focus of the microterminal is objective item testing in the form of embedded or adjunct questions accompanying instruction and in the form of end-ofinstruction achievement tests. The hardware/software design of the first prototype unit focused on a serial sequence of test items, with flexibility for control of feedback, response storage, and the retrieval of student response data.

\section{HARDWARE}

The external hardware (Figure 1) interacts with a student via a 16-key keyboard, four hexidecimal displays, and 14 light-emitting diodes (LEDs) associated with various messages for student control. Only 12 of the keys are used for student interaction. Three are special-purpose keys used by an instructor in a special instructor mode for manual data retrieval. Keys are labeled 0 through 9, SEND, and CLEAR, with Keys 1 through 5 serving dual functions, incorporating the question response letters $\mathrm{A}$ through $\mathrm{E}$.

The four hexidecimal displays each project a 5 by 7 dot array, depicting a number from 0 through 9 or a letter from A through $F$. These displays afford visual keypress feedback to the student as well as question number and response item representation.

Fourteen miniature LEDs, associated with various messages, direct the student through a testing sequence. These messages are: ENTER STUDENT IDENTIFICATION NUMBER; ENTER BOOKLET NUMBER; ANSWER THIS QUESTION; YOUR SCORE IS; ELAPSED TIME: HRS.-MINS.; MISSED QUESTION AND RESPONSE; INSTRUCTOR MODE; RETAKE MISSED QUESTION(s); SEE YOUR INSTRUCTOR; PRESS SEND; YES; and NO. Currently only 12 of these LEDs are used.

External hardware is under the control of a Motorola M6800 microprocessor chip and its related family of part members consisting of a $1-\mathrm{MHz}$ clock chip for system timing requirements, an MCM6820 peripheral interface adapter (PIA) chip for parallel communication to external hardware, and two MCM6810L-1 static random-access-memory (RAM) chips for storage of student data. The two RAM memories give a total capacity for data storage of 2568 -bit words. This is more than enough for student sign-on data and test response storage.

The microterminals main control program is stored in six Intel Corporation 2704 reprogrammable readonly-memory (PROM) chips, compatible to the Motorola M6800, but a nonfamily part. These chips 


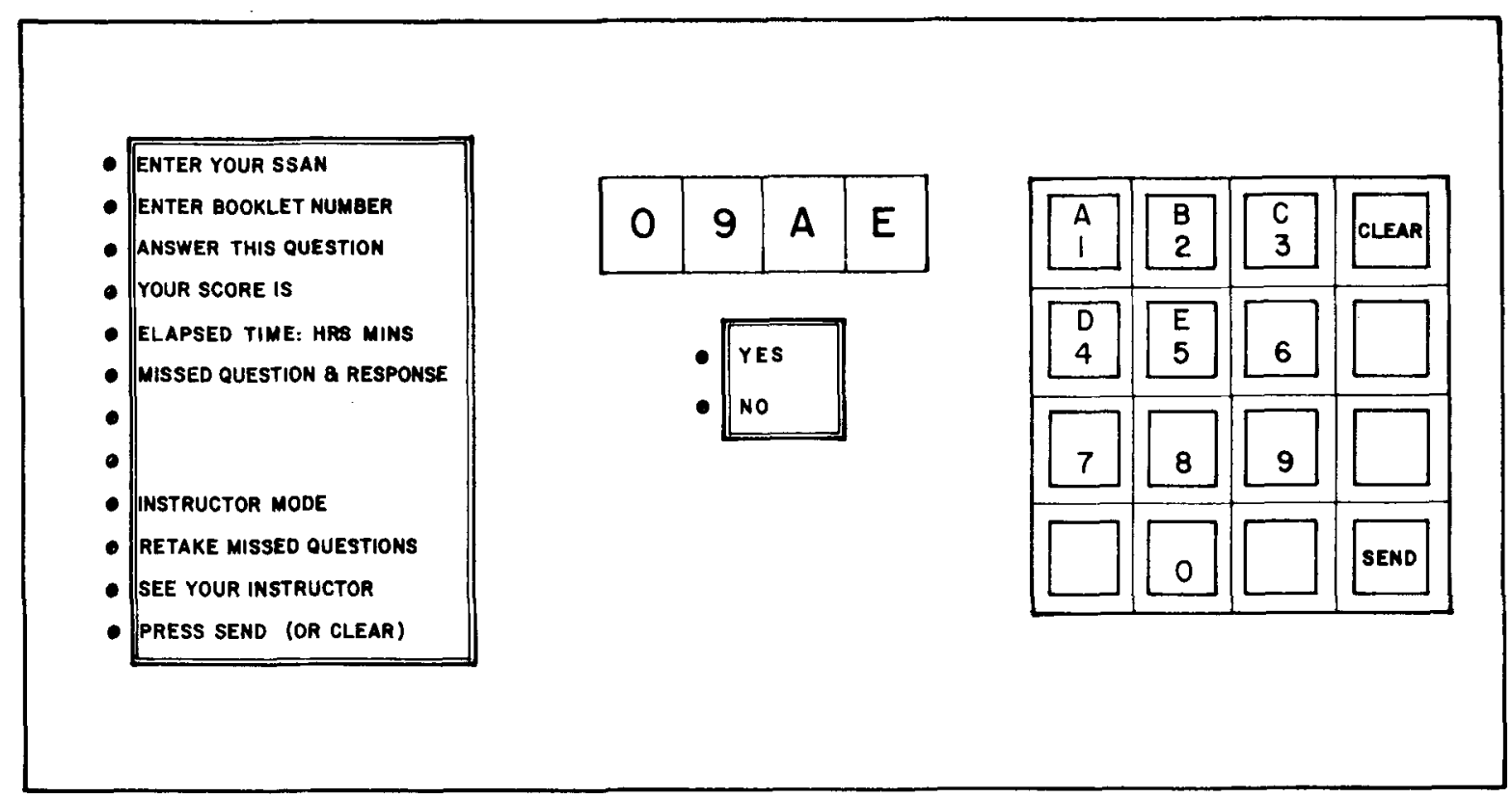

Figure 1. Microterminal faceplate.

each contain 512 8-bit words, for a total memory storage capacity of over 3,000 8-bit words. Reprogrammable chips are ideally suited for developing prototype equipment.

Various other compatible but non-Motorola devices are used as required for system interface and buffering to the displays, LEDs, and keyboard. An external, triple-output power supply is used with the prototype microterminal; the three voltages are required by the 2704 PROM chips. This supply, when enclosed within the microterminal case, along with rechargeable batteries for memory data retention, allows the microterminal to be a completely portable device.

All hardware components, both external and internal to the microterminal, are off-the-shelf components affording easy maintenance and fast turnaround should a hardware failure occur. The M6800 is the heart of our system because of its hardware/software design ease, its requirement for only a single $5 \cdot \mathrm{V}$ power supply, and its availability at the start of this project with ample hardware/software design documentation.

Hardware costs for the prototype are less than $\$ 400$ per unit, a price that does not reflect actual costs for a production model device. Optimizing circuit design, defining actual hardware/software requirements, and quantity pricing could greatly reduce the unit price. Hardware cost savings are being realized almost monthly by price reductions being made on microprocessor and memory components.

\section{SOFTWARE}

The software of the microterminal can best be described in terms of a student/microterminal testing scenario as follows:
A student acquires a microterminal at a test center, takes the device to a noncomputerized student testing carrel, plugs the device into power, and follows the directions given by the LEDs and their associated messages. Under software control the student is first directed to enter a student identification number. Since this number consists of nine digits, it is presented to the student four digits at a time as it moves across the display. After entering this number, the student is directed by a message LED to press SEND. At some time before he presses SEND, the option exists to clear the response by pressing the CLEAR key, allowing the student to enter a new series of numbers. This function holds true throughout the test, affording the capability to clear a response at any time prior to pressing SEND. After pressing SEND, the student identification number

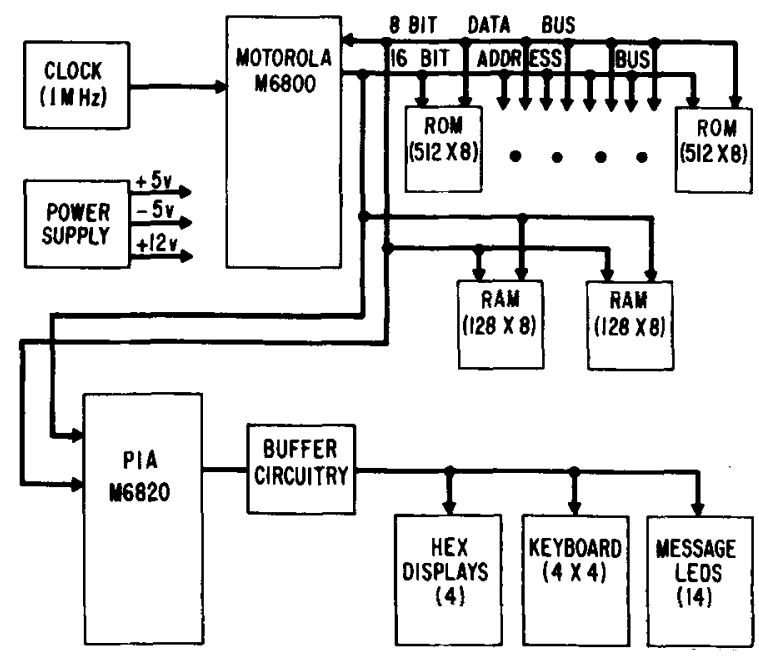

Figure 2. Microterminal block diagram. 
is accepted and stored in RAM and the LED lights, directing the student to ENTER BOOKLET NUMBER. After properly entering this number, the press SEND light illuminates, directing the student to again press SEND.

The booklet number the student enters consists of five numbers, decoded by the software, and allows the selection of various testing options. The first digit is a number from 1 to 4 , and depending on the number entered, directs the student to one of four independent test strategies. If a 1 is entered, the student takes a test without YES or NO feedback, representative of an endof-instruction test. A 2 allows for YES/NO feedback and directs a student straight through a review of material type test with immediate feedback. The third strategy administers a test that makes the student remain at a missed question until properly answered before displaying the next question. The fourth strategy is almost the same as the third except for the addition of a loopback feature, forcing the student to go back through a list of missed questions giving multiple chances at items that seem to be presenting problems. These four strategies are not all-inclusive and can be expanded upon by changing software and/or hardware features of the microterminal

The next two digits of the booklet riumber locate one of 30 preprogrammed test answer keys used to evaluate student responses. The 30 test keys contain 30 answers each, providing a total answer bank of 900 items. The maximum number of items per test for our testing requirements is 30 , although with different testing algorithms it is possible to increase this amount up to 900 if needed.

The last two digits of the booklet number are variable from 1 to 30 and are the number of test items. This number is used to compute the score at the end of the test for retrieval by the instructor or for input into the management computer system.

After proper entry of the student booklet number, the student is again directed by a third LED message light which instructs the student to ANSWER THIS QUESTION. The question number is displayed on one or two of the leftmost hexidecimal displays. The student is then allowed to respond to the item by pushing a letter from $A$ to $E$ on the keyborad. The letter response is displayed on the rightmost display. When the student is satisfied that the response is correct, he presses the SEND key, and depending on the preselected strategy, receives or does not receive YES/NO feedback and then proceeds to the next question. When the student completes the test, the device is hand carried to an on-line interface terminal to dump the accumulated data into the main computer system. It takes less than $1 / 2 \mathrm{sec}$ for the data to be transferred, and within a short period of time, test score feedback and further prescription information are available to the student at the printer.

The software package for the microterminal handles several features normally associated with hardware. Student test time is monitored by the microterminal in hours and minutes and can be displayed when in instructor mode. Key debounce is accomplished by the software, along with various decoding functions for the displays and keyboard.

Access to instructor mode, which would be a classified keying arrangement in regard to the student, allows the instructor to retrieve the student identification number, booklet number, items missed, test time, and score. This secondary mode of operation extends the capability of the microterminal to act as a stand-alone device and, in effect, to become its own output for feedback of test results.

\section{MICROTERMINAL EVALUATION}

A pilot study of a prototype microterminal was performed to verify the usability and human factors of the design and to attempt to gain a preliminary insight into potential instructional effects of the device. Results of the pilot study showed high user acceptance of the device, with no significant test-grade differences between users and nonusers. The study indicated that students who used the microterminal accomplished lesson materials $30 \%$ faster than did the control group. The time results of the study are shown in Table 1 . The difference in times between the groups may be due to a combination of factors, for example, time to fill out computer answer sheets, effect of a computer directly monitoring the student's progress, increased attending behavior due to the direct tutorial effect of using the responder unit, or simply a Hawthorne effect.

An attitude questionnaire was administered to elicit student reactions to the device. The attitude questionnaire and student responses (percent) are shown in Table 2. Three items in particular seem important for systems design. Item 2 indicates that students prefer a device rather than a form for recording their responses. Item 12 shows that a computer terminal device can be built to control the response feedback situation and give clear directive-type information without an elaborate computer display. In other words, the device satisfied an important part of the instructional situation without having to pay for unused features such as a large display, due to the information load being carried by the pro-

Table 1

Lesson Completion Times

\begin{tabular}{|c|c|}
\hline Control & Microterminal \\
\hline $\begin{array}{l}\mathrm{N}=12 \\
\bar{X}_{1}=168.08 \mathrm{~min} \\
S D=80.5\end{array}$ & $\begin{array}{l}\mathrm{N}=12 \\
\overline{\mathrm{X}}_{2}=117.75 \mathrm{~min} \\
\mathrm{SD}=50.5\end{array}$ \\
\hline \multicolumn{2}{|c|}{$\begin{array}{c}\overline{\mathrm{X}}_{1}-\overline{\mathrm{X}}_{2}=50.33 \mathrm{~min} \\
\mathrm{t}=1.85, \text { significant at } .05 \text { (one-tailed) }\end{array}$} \\
\hline
\end{tabular}


Table 2

Questionnaire Results of Student's Acceptance of the Microterminal

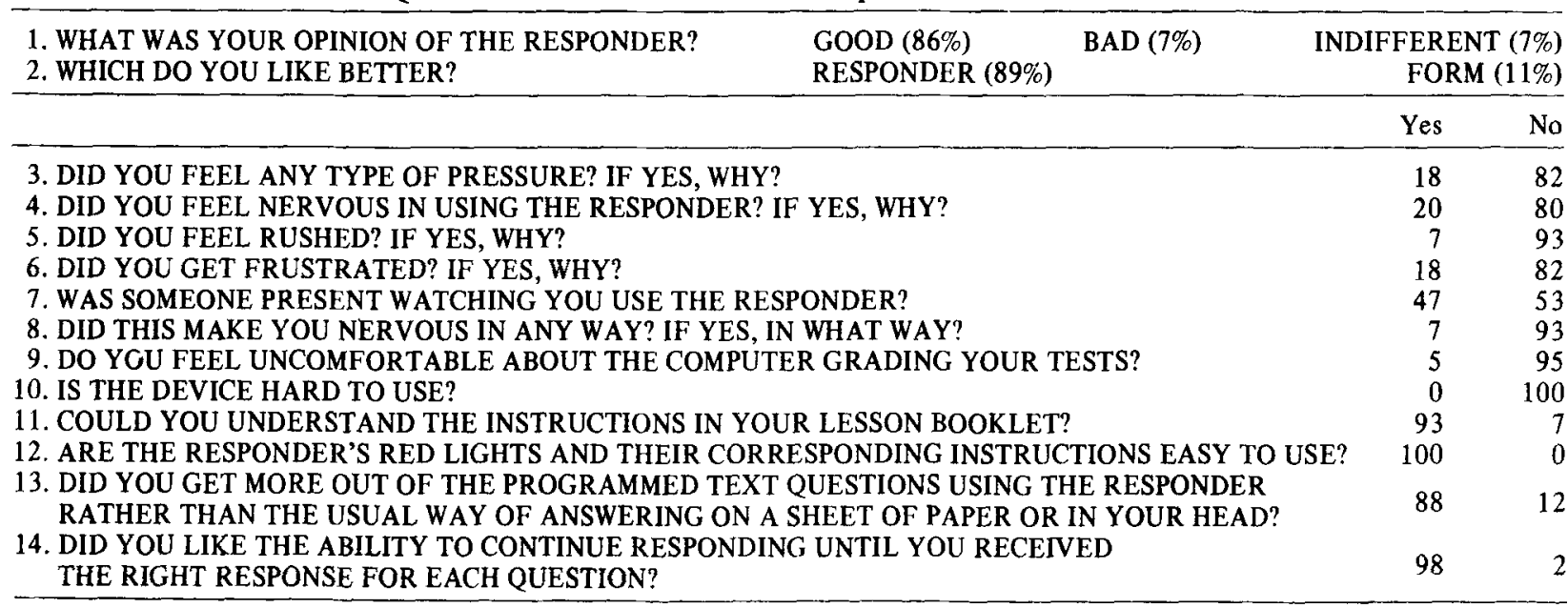

Note $-N=41$. Numbers represent percentages.

grammed text on the test itself. Item 13 is of particular interest. Students subjectively reported that they got more out of the responder than if they used a sheet of paper to answer embedded questions. Normally, students are simply directed to record their answers on a sheet of paper, with feedback being provided in back of the programmed text. The microterminal has an advantage because the student must commit himself to an answer before it is possible to receive feedback.

The results of any pilot study require more extensive confirmation; however, the present results are reported because they adequately represent the viability of a particular hardware solution to an identified instructional need in a computer-based instructional system. The instructional implications of the use of a microterminal as described in this report can be significant. In the area of testing, the use of different testing strategies, test item security, and automated entry of test results for item or response analysis by a larger system become administratively feasible or simpler to implement.

\section{FUTURE DEVELOPMENT}

The prototype microterminal described in this paper is in no way an optimized version of a testing device. One of our design goals was to simply incorporate the use of a few selected testing strategies into a microcomputer-controlled testing device and to test the feasibility of such a device as a replacement to standard paper-and-pencil tests. With the phenomenal advances being made in state-of-the-art microprocessors and memory chips, our microterminal can be redesigned to be more compact, lower in cost, use less power, with a greater yield in capability.

Later versions of the microterminal will incorporate the use of the M6802, a third-generation microprocessor that incorporates its own clock circuitry and 1,000 words of RAM within the processor chip itself. Other processors are being marketed with PROM also on board, so that in effect a microprocessor becomes a complete and self-contained computer system with associated memory in a small 40-pin package.

Two new Texas Instruments TMS2716 PROMs will replace the six 2704 PROMs in our microterminal. This not only decreases cost with an increase in maximum storage capacity, but also cuts power requirements and decreases printed circuit board size.

If more variable storage (RAM) is needed (possibly due to an increase in test size), higher density, lower power, and less expensive RAM chips could replace the present MCM6810L-1 static RAM chips now used. We have chosen to replace our RAM memory with two Intersil 6561 low-power RAM chips which will reside on a small memory module that actually plugs in to the microterminal case. This module will be powered by two Eveready B50T batteries which supply enough power to allow data retention for a couple of weeks without being recharged and will also retain data should there be a power failure when a student is taking a test.

A substantial savings in power and an increase in versatility has been accomplished by replacing the four hexidecimal displays with a single-package low-power alphanumeric display. With this display, it is now possible for us to introduce single-word instructions to the student via the display, although on a very limited basis. An example might be to print the word "DONE" when all questions to a particular test have been completed.

We are continually expanding our efforts in investigating extended microterminal applications that can be derived by adding input/output communication links to a microterminal. These links would be capable of controlling any number of media equipment, such as projectors, microfiche devices, etc. 


\section{SUMMARY}

This simple microcomputer-controlled testing device and the associated software routine has eliminated the paper-and-pencil objective-type test and allowed for a wide variety of testing strategies. Data retrieval can be accomplished at a slow manual rate in instructor mode or at high speed through the use of the management system. Student operation of the device is quickly learned and needs little, if any, introductory instruction. After an initial burn-in period, which is characteristic of all electronics equipment, the device should operate maintenance free for many hours. The prototype has operated without any failure approximately 8 to $10 \mathrm{~h} /$ day, 5 days a week, in excess of 60 weeks. 